\title{
Electric-field-induced shift of the Mott metal-insulator transition in thin films
}

\author{
D. Nasr Esfahani, L. Covaci, and F. M. Peeters \\ Departement Fysica, Universiteit Antwerpen, Groenenborgerlaan 171, B-2020 Antwerpen, Belgium \\ (Received 5 September 2011; revised manuscript received 3 January 2012; published 14 February 2012)
}

\begin{abstract}
The ground-state properties of a paramagnetic Mott insulator at half-filling are investigated in the presence of an external electric field using the inhomogeneous Gutzwiller approximation for a single-band Hubbard model in a slab geometry. We find that the metal-insulator transition is shifted toward higher Hubbard repulsions by applying an electric field perpendicular to the slab. The main reason is the accumulation of charges near the surface. The spatial distribution of site-dependent quasiparticle weight shows that it is maximal in a few layers beneath the surface, while the central sites where the field is screened have a very low quasiparticle weight. Our results show that above a critical-field value, states near the surface will be metallic, while the bulk quasiparticle weight is extremely suppressed but never vanishing, even for large Hubbard repulsions above the bulk zero-field critical value. Below the critical-field value, our results hint toward an insulating state in which the electric field is totally screened and the slab is again at half-filling.
\end{abstract}

DOI: 10.1103/PhysRevB.85.085110

PACS number(s): 71.30.+h, 71.27.+a, 73.61. $-\mathrm{r}$

\section{INTRODUCTION}

The rich physics of strongly correlated materials in combination with the need to overcome the scaling limits of current silicon-based semiconductor materials in the microelectronic industry has resulted in an increased activity in this field. Special attention has been focused on vanadium dioxide $\left(\mathrm{VO}_{2}\right)$, which shows an abrupt metal-insulator transition (MIT) near room temperature due to a structural phase transition. ${ }^{1}$ It was found that an electric field is also able to trigger MIT in $\mathrm{VO}_{2}$, without any structural transition, which is mostly dominated by electron correlations rather than a Peierls distortion. ${ }^{2}$ Also, a first-order MIT is observed by applying an electric field in a two-terminal model of $\mathrm{VO}_{2}$ (Refs. 3 and 4) without electrical breakdown of the material. Note that an electric-field-driven MIT and metal-superconductor transitions have been observed at the interface between $\mathrm{LaAlO}_{3}$ and $\mathrm{SrTiO}_{3}{ }^{3}$ These kinds of transitions may be related to a charge transfer mechanism. A nonlinear dependence of the conductivity on the electric field is reported for the highly correlated transition-metal chalcogenide $\mathrm{Ni}(\mathrm{S}, \mathrm{Se})_{2}$, and a continuous MIT is observed in this case. ${ }^{5}$

In this paper, we investigate the behavior of the ground state of a single-band Hubbard model ${ }^{6}$ in the presence of an electric field by using the Gutzwiller approximation (GA). ${ }^{7}$ Originally, GA is rooted in the Gutzwiller wave function used to reduce the contribution of high-energy states due to Hubbard repulsion and it was shown to be exact in the limit of infinite dimensions. ${ }^{8-10}$

While an analytical solution exists only for one dimension, ${ }^{11}$ in comparison to other approximate methods, the GA is equivalent to a slave-boson mean-field ${ }^{12}$ (SBMF) approach for zero temperature, but in contrast to dynamical mean-field theory (DMFT), ${ }^{13}$ it is not able to give any information about higher and lower Hubbard bands. Instead, it gives a reasonable understanding about the low-energy excitations near the Fermi surface ${ }^{14}$ by supplying the quasiparticle (QP) weight of electrons such that one is then able to describe the mobility of electrons. Also, GA can not give any information about the insulating state, instead we are only able to investigate the properties of the system by approaching the transition point $U_{c}$ from below. ${ }^{10}$ This method was used by Brinkman and Rice ${ }^{15}$ to investigate the MIT of the single-band Hubbard model and it allowed them to predict the critical Hubbard repulsion, which is finite in two and three dimensions $\left(U_{c}=16 t\right.$ for $\left.3 \mathrm{D}\right)$. While not as accurate as DMFT, GA is less computationally intensive and thus allows the description of inhomogeneous systems such as thin films subjected to a perpendicular electric field.

Although our simplified approach is only qualitative, it gives important information about how one may be able to spatially tune the quasiparticle weight distribution near surfaces and interfaces. This could be relevant for future studies; for example, for an inhomogeneous bad metal-superconductor transition by the charge transfer mechanism, which may be responsible for the superconductor (SC) insulator transition observed at the interface of a band insulator and a strongly correlated material. ${ }^{3}$ We will show that by applying a perpendicular electric field, charges will be trapped at the surface of the Mott insulator and shift the MIT for the surface states.

The outline of the paper is as follows. In Sec. II, we review the concept of GA and discuss the extension of the method to include the effect of onsite potentials. In Sec. III, we introduce our model for the slab geometry, present the numerical scheme used, and analyze the corresponding results. Finally, in Sec. IV, we present our conclusions.

\section{GUTZWILLER APPROXIMATION IN THE PRESENCE OF AN ELECTRIC FIELD}

In order to address the narrow band effects in transition metals with $d$ or $f$ orbitals for which correlation effects play a major role in the behavior of the system, the simplest model that is able to explain the most important terms of the Coulomb interaction between electrons is the well-known Hubbard model

$$
\hat{H}_{U}=-\sum_{\langle i j\rangle \sigma} t_{i j}\left(c_{i \sigma}^{\dagger} c_{j \sigma}+c_{j \sigma}^{\dagger} c_{i \sigma}\right)+\sum_{i} U \hat{n}_{i \sigma} \hat{n}_{i \bar{\sigma}} .
$$

We will describe the ground-state properties of the Hubbard model by using the Gutzwiller approximation, which suppresses the contribution of high-energy states (these are 
configurations with a higher number of double occupancies). This is done by introducing a trial wave function, which contains variational parameters that are subsequently used to minimize the total energy of the system. Our aim is to investigate the properties of a strongly correlated system in the presence of an external electric field modeled in the Hamiltonian as a position-dependent potential in the field direction, while translational invariance is maintained in the direction perpendicular to the applied field. To study the ground-state properties in the absence of the electric field, the Gutzwiller wave function is defined as

$$
\left|\psi_{g}\right\rangle=\prod_{i} g_{i}^{\hat{D}_{i}}\left|\psi_{0}\right\rangle=\prod_{i}\left[1-\left(1-g_{i}\right) \hat{D}_{i}\right]\left|\psi_{0}\right\rangle,
$$

where the double occupancy operator is $\hat{D}_{i}=\hat{n}_{i \sigma} \hat{n}_{i \bar{\sigma}}$, the variational parameters $g_{i}$ are introduced to reduce the contribution of high-energy configurations in the many-body wave function $\left|\psi_{g}\right\rangle$, and $\left|\psi_{0}\right\rangle$ is the unprojected noninteracting (Fermi sea) many-body wave function. Although it is obvious that by the inclusion of onsite potentials, no new variational parameters are needed because they do not induce any new correlations since the term is a single-body interaction, nevertheless we will prove it rigorously. To obtain the normalization factors in the limit of spatial infinite dimensions, for which the Gutzwiller approximation is exact, ${ }^{9,10}$ we have to remove spatial correlations that occur in infinite dimensions together with onsite Hartree contributions, which remain in the $d=\infty$ limit. This can be done by introducing an expansion parameter following the guidelines of Ref. 10. If we include onsite potentials for capturing the effects of external fields, the Hamiltonian becomes

$$
\hat{H}=\hat{H}_{U}+\sum_{i \sigma} v_{i} \hat{n}_{i \sigma} .
$$

In order to find the ground state of the Hamiltonian in Eq. (3), we introduce variational parameters $\zeta_{i \sigma}$ and $\zeta_{i \bar{\sigma}}$ to decrease the weight of the occupancy of the sites with higher onsite energy. The Gutzwiller wave function now becomes

$$
\begin{aligned}
\left|\psi_{g}\right\rangle= & {\left[1-\left(1-\zeta_{i \sigma}\right) \hat{n}_{i \sigma}\right]\left[1-\left(1-\zeta_{i \bar{\sigma}}\right) \hat{n}_{i \bar{\sigma}}\right] } \\
& \times\left[1-\left(1-g_{i}\right) \hat{D}_{i}\right]\left|\psi_{0}\right\rangle .
\end{aligned}
$$

The standard way of removing onsite Hartree contributions is to introduce the fugacity factors $\mu_{i \sigma}$ and $\mu_{i \bar{\sigma}},{ }^{10}$ the expansion parameter $x_{i}$, and the noninteracting state $\left|\varphi_{0}\right\rangle$. Then, the Gutzwiller wave function can be written as

$$
\begin{aligned}
\left|\psi_{g}\right\rangle & =\prod_{i} \zeta_{i \sigma}^{\hat{n}_{i \sigma}} \zeta_{i \bar{\sigma}} \hat{n}_{i \bar{\sigma}} g_{i}^{2\left(\gamma_{i}-\mu_{i \bar{\sigma}} \hat{n}_{i \bar{\sigma}}-\mu_{i \sigma} \hat{n}_{i \sigma}+\hat{D}_{i}\right)}\left|\varphi_{0}\right\rangle \\
& =\prod_{i}\left(1+x_{i}\left(\hat{D}_{i}-\hat{D}_{i}^{H F}\right)\right)\left|\varphi_{0}\right\rangle .
\end{aligned}
$$

The Hartree double occupancy operator can be defined as $\hat{D}_{i}^{H F}=\hat{n}_{i \sigma}\left\langle\hat{n}_{i \bar{\sigma}}\right\rangle_{0}+\left\langle\hat{n}_{i \sigma}\right\rangle_{0} \hat{n}_{i \bar{\sigma}}-\left\langle\hat{n}_{i \sigma}\right\rangle_{0}\left\langle\hat{n}_{i \bar{\sigma}}\right\rangle_{0}$ and it is the result of the usual mean-field decomposition $\hat{n}_{i \sigma} \rightarrow \hat{n}_{i \sigma}-$ $\left\langle\hat{n}_{i \sigma}\right\rangle_{0}$. By defining $\zeta_{i \sigma}=g_{i}{ }_{i \sigma}, \zeta_{i \bar{\sigma}}=g_{i}{ }^{\beta_{i \bar{\sigma}}}, \mu_{i \sigma}{ }^{\prime}=\beta_{i \sigma}+\mu_{i \sigma}$, and $\mu_{i \bar{\sigma}}{ }^{\prime}=\beta_{i \bar{\sigma}}+\mu_{i \bar{\sigma}}$, we have

$$
\left|\psi_{g}\right\rangle=\prod_{i} g_{i}^{2\left(\gamma_{i}-\mu_{i \bar{\sigma}}{ }^{\prime} \hat{n}_{i \bar{\sigma}}-\mu_{i \sigma}{ }^{\prime} \hat{n}_{i \sigma}+\hat{D}_{i}\right)}\left|\varphi_{0}\right\rangle .
$$

Therefore, by using the above change of variables, it is possible to obtain the same renormalization factors for the infinite dimensions limit as stated in Ref. 10. Moreover, by using the condition $\left\langle\hat{n}_{i \sigma}\right\rangle=\left\langle\hat{n}_{i \sigma}\right\rangle_{0}$, which holds for infinite dimensions, it can be inferred that the physical counterparts of the variational parameters $\zeta_{i \sigma}$ are $\left\langle\hat{n}_{i \sigma}\right\rangle_{0}$. We need to minimize the energy with respect to $\left|\varphi_{0}\right\rangle$ together with local variational parameters $g_{i}$ needed to describe the correlation effects. In short, the addition of onsite potentials does not add any new variational parameters and the procedure of finding the ground state is the same as in the conventional Gutzwiller method. Thus, the expectation value of the Hamiltonian

$$
\begin{aligned}
\langle\hat{H}\rangle= & -\sum_{\langle i j\rangle \sigma} \sqrt{q_{i \sigma}} \sqrt{q_{j \sigma}} t_{i j}\left\langle\varphi_{0}\right| \hat{c}_{j \sigma}^{\dagger} \hat{c}_{i \sigma}+\text { H.c. }\left|\varphi_{0}\right\rangle \\
& +\sum_{i} V_{i}\left\langle\varphi_{0}\left|\hat{n}_{i}\right| \varphi_{0}\right\rangle+\sum_{i} U \bar{d}_{i}
\end{aligned}
$$

has to be minimized only with respect to $g_{i}$ and $\left|\varphi_{0}\right\rangle$. Here, the renormalization factors $q_{i \sigma}$ depend on the local density of the noninteracting state $\left|\varphi_{0}\right\rangle$ and $g_{i}$ :

$$
\begin{aligned}
q_{i \sigma}= & \frac{1}{\left\langle\hat{n}_{i \sigma}\right\rangle_{0}\left(1-\left\langle\hat{n}_{i \sigma}\right\rangle_{0}\right)}\left[\sqrt{d_{i}\left(\left\langle\hat{n}_{i \sigma}\right\rangle_{0}-d_{i}\right)}\right. \\
& \left.+\sqrt{\left(\left\langle\hat{n}_{i \bar{\sigma}}\right\rangle_{0}-d_{i}\right)\left(1-n_{i, 0}+d_{i}\right)}\right]^{2}
\end{aligned}
$$

where $n_{i, 0}=\left\langle\hat{n}_{i \sigma}\right\rangle_{0}+\left\langle\hat{n}_{i \bar{\sigma}}\right\rangle_{0}$, while $g_{i}$ are described by the following equations, which hold in infinite dimensions:

$$
g_{i}^{2}=\frac{d_{i}\left(1-n_{i, 0}+d_{i}\right)}{\left(\left\langle\hat{n}_{i \bar{\sigma}}\right\rangle_{0}-d_{i}\right)\left(\left\langle\hat{n}_{i \sigma}\right\rangle_{0}-d_{i}\right)} .
$$

Although in normal metals any deviation from half-filling may lead to the lowering of the electron conductivity, in strongly correlated materials, doping plays a different role because of the dependence of tight-binding renormalization factors $q_{i \sigma}$ on the local charge density. Thus, one may predict that if an applied electric field would be able to change the charge distribution of the system, then it will be able to change the electron conductivity and even shift the metal-insulator transition point.

In practice, minimizing the expectation value of the Hamiltonian is difficult because of the existence of a large number of variational parameters in $\left|\varphi_{0}\right\rangle$ together with the dependence of the renormalization factors on $\left|\varphi_{0}\right\rangle$. This will lead to a highly nonlinear set of equations. In order to alleviate some of the difficulties, it is possible to allow local densities and $\left|\varphi_{0}\right\rangle$ to vary independently in the minimization procedure. Then, by introducing the Lagrange multipliers $\lambda_{i \sigma}$, it is possible to ensure that the local charge densities of the Gutzwiller wave function are equal to the local charge densities of the noninteracting state. Other multipliers, $\Lambda$ and $E$, are introduced in order to ensure total charge conservation and guarantee that $\left|\varphi_{0}\right\rangle$ is normalized. Therefore, the final form of the energy expectation value is

$$
\begin{aligned}
\langle\hat{H}\rangle= & -\sum_{\langle i j\rangle \sigma} \bar{t}_{i j}\left\langle\varphi_{0}\right| \hat{c}_{j \sigma}^{\dagger} \hat{c}_{i \sigma}+\text { H.c. }\left|\varphi_{0}\right\rangle \\
& +\sum_{i, \sigma} v_{i}\left\langle\varphi_{0}\left|\hat{n}_{i \sigma}\right| \varphi_{0}\right\rangle+\sum_{i} U \bar{d}_{i}
\end{aligned}
$$




$$
\begin{aligned}
& +\sum_{i, \sigma} \lambda_{i \sigma}\left(\left\langle\hat{n}_{i \sigma}\right\rangle_{0}-n_{i \sigma}\right) \\
& +\Lambda\left(N-\sum_{i \sigma} n_{\sigma i}\right)+E\left(1-\left\langle\varphi_{0} \mid \varphi_{0}\right\rangle\right),
\end{aligned}
$$

where $\bar{t}_{i j}=\sqrt{q_{i \sigma}} \sqrt{q_{j \sigma}} t_{i j}$ are the renormalized hopping amplitudes. To find the optimum energy, we first vary the $\left|\varphi_{0}\right\rangle$, which obeys the following Schrödinger-type equation:

$$
\begin{aligned}
& \sum_{\langle i j\rangle \sigma}-\bar{t}_{i j}\left(\hat{c}_{j \sigma}^{\dagger} \hat{c}_{i \sigma}+\text { H.c. }\right)\left|\varphi_{0}\right\rangle \\
& \quad+\sum_{i}\left(V_{i}+\lambda_{i \sigma}\right) \hat{n}_{i \sigma}\left|\varphi_{0}\right\rangle=E\left|\varphi_{0}\right\rangle,
\end{aligned}
$$

which is solved by exact diagonalization for both spins. This noninteracting energy is the amount of kinetic energy that is stored in the QP state $\left|\varphi_{0}\right\rangle$. Then, $\left|\varphi_{0}\right\rangle$ is substituted in Eq. (10) and the expectation value becomes

$$
\langle\hat{H}\rangle=E_{N I}+\sum_{i} U \bar{d}_{i}+\Lambda\left(N-\sum_{i, \sigma} n_{i \sigma}\right)+\sum_{i, \sigma} \lambda_{i \sigma} n_{i \sigma},
$$

where $E_{N I}$ is the noninteracting energy, which depends on the variational parameters $n_{i \sigma}, \lambda_{i \sigma}$, and $\left|\varphi_{0}\right\rangle .\left|\varphi_{0}\right\rangle$ is now a function of the variational parameters $\lambda_{i \sigma}, n_{i \sigma}$, and $g_{i}$, and the above energy functional has to be minimized in accordance to all these parameters. This leads to the following set of saddle-point conditions:

$$
\begin{array}{ll}
\frac{\partial\langle\hat{H}\rangle}{\partial \Lambda}=0, & \frac{\partial\langle\hat{H}\rangle}{\partial \lambda_{i \sigma}}=0, \\
\frac{\partial\langle\hat{H}\rangle}{\partial n_{i \sigma}}=0, & \frac{\partial\langle\hat{H}\rangle}{\partial g_{i}}=0 .
\end{array}
$$

In general, the onsite potential profile is a functional of the spatial density distribution due to the effects of long-range electron-electron and electron-ion interactions. This effect should be addressed by considering an additional Poisson equation in the set of equations. Although the effect of charge redistribution due to long-range interactions has major effects in weak Hubbard coupling, it has minor effects in intermediate- and strong-coupling regimes due to the already very large screening effects induced by the Hubbard interaction at half-filling.

\section{MODEL AND NUMERICAL SCHEME}

\section{A. Model}

We consider a slab geometry with translational invariance in the $x$ and $y$ directions and finite size in the $z$ direction. In addition, we apply a linear potential profile from $-v / 2$ to $+v / 2$ in the $z$ direction.
With the above assumptions, the expectation value of the Hamiltonian can be written as

$$
\begin{aligned}
\langle\hat{H}\rangle= & \left\langle\varphi_{0}\right| \sum_{i, k_{\|}, \sigma}\left[-2 t q_{i \sigma}\left(\cos k_{x}+\cos k_{y}\right)+v_{i}+\lambda_{i \sigma}\right] \hat{c}_{i k_{\|} \sigma}^{\dagger} \hat{c}_{i k_{\|} \sigma} \\
& -\sum_{\langle i j\rangle k_{\|} \sigma} \sqrt{q_{i \sigma}} \sqrt{q_{j \sigma}} t\left(\hat{c}_{i k_{\|} \sigma}^{\dagger} \hat{c}_{j k_{\|} \sigma}+\hat{c}_{j k_{\|} \sigma}^{\dagger} \hat{c}_{i k_{\|} \sigma}\right)\left|\varphi_{0}\right\rangle \\
& -N_{k_{\|}} \sum_{i \sigma} \lambda_{i \sigma} n_{i \sigma}+\Lambda\left(N_{k_{\|}} \sum_{i \sigma} n_{i \sigma}-N\right) \\
& \left.+E\left(1-\left\langle\varphi_{0}\right| \varphi_{0}\right)\right\rangle+\sum_{i} N_{k_{\|}} U d_{i},
\end{aligned}
$$

where $i$ and $j$ correspond to atoms in the $z$ direction and $N_{k_{\|}}=N_{k_{x}} N_{k_{y}}$ is the total number of $k$ points.

First, we minimize the energy with respect to $\left|\varphi_{0}\right\rangle$, which leads to the following eigenvalue problem:

$$
\begin{aligned}
& \sum_{i, \sigma}\left[-2 t q_{i \sigma}\left(\cos k_{x}+\cos k_{y}\right)+v_{i}+\lambda_{i \sigma}\right] \\
& \times \hat{c}_{i k_{\|} \sigma}^{\dagger} \hat{c}_{i k_{\|} \sigma}\left|\varphi_{0}\right\rangle-\sum_{\langle i j\rangle \sigma} \sqrt{q_{i \sigma}} \sqrt{q_{j \sigma}} t \\
& \quad \times\left(\hat{c}_{i k_{\|} \sigma}^{\dagger} \hat{c}_{j k_{\|} \sigma}+\hat{c}_{j \sigma}^{\dagger} \hat{c}_{i \sigma}\right)\left|\varphi_{0}\right\rangle=E_{k_{\|}}\left|\varphi_{0}\right\rangle .
\end{aligned}
$$

Equation (15) has to be solved for each $k_{\|}$point, and in order to find the noninteracting ground state, the eigenvalues will be summed up to the desired filling level:

$$
E_{N I}=\sum_{E<E_{F}} E_{k_{\|}, n},
$$

where $E_{F}$ is the Fermi energy of the QP states and $n$ is the quantum number for the energy level of each $k$ point.

In the next step, the above noninteracting state $\left|\varphi_{0}\right\rangle$, which is now an implicit function of all variational parameters $\lambda_{i \sigma}$, $n_{i \sigma}, g_{i}$, and $\Lambda$, should be inserted into Eq. (14):

$$
\begin{aligned}
\langle\hat{H}\rangle= & \left\langle\varphi\left[\lambda_{i \sigma}, n_{i \sigma}, g_{i}, \Lambda\right]\left|\hat{H}_{0}\right| \varphi\left[\lambda_{i \sigma}, n_{i \sigma}, g_{i}, \Lambda\right]\right\rangle \\
& +N_{k_{\|}} \sum_{i, \sigma} \lambda_{i \sigma} n_{i \sigma}+\Lambda\left(N_{k_{\|}} \sum_{i, \sigma} n_{i \sigma}-N\right) .
\end{aligned}
$$

We therefore minimize the total energy according to the variational parameters by considering

$$
\frac{\partial}{\partial \lambda}\left\langle\psi[\lambda]\left|H_{0}[\lambda]\right| \psi[\lambda]\right\rangle=\left\langle\psi[\lambda]\left|\frac{\partial}{\partial \lambda} H_{0}[\lambda]\right| \psi[\lambda]\right\rangle,
$$

which holds when the wave function is an eigenfunction of the noninteracting Hamiltonian and obtain the following set of saddle-point equations for the paramagnetic case $\left(\left\langle\hat{n}_{i \sigma}\right\rangle=\right.$ $\left.\left\langle\hat{n}_{i \bar{\sigma}}\right\rangle\right)$ :

$$
\begin{aligned}
\frac{\partial\langle\hat{H}\rangle}{\partial g_{i}}= & 2\left\langle\varphi_{0}\right| \sum_{i k_{\|}}-2 t\left(\cos k_{x}+\cos k_{y}\right) \frac{\partial q_{i \sigma}}{\partial g_{i}} \hat{c}_{i k_{\|} \sigma}^{\dagger} \hat{c}_{i k_{\|} \sigma} \\
& -\delta_{i, j \pm 1} \sqrt{\frac{q_{j \sigma}}{q_{i \sigma}}} \frac{\partial q_{i \sigma}}{\partial g_{i}} t\left(\hat{c}_{i k_{\|} \sigma}^{\dagger} \hat{c}_{j k_{\|} \sigma}+\hat{c}_{j k_{\|} \sigma}^{\dagger} \hat{c}_{i k_{\|} \sigma}\right)\left|\varphi_{0}\right\rangle \\
& +\sum_{i} N_{k_{\|}} U \frac{\partial d_{i}}{\partial g_{i}}=0
\end{aligned}
$$




$$
\begin{gathered}
\frac{\partial\langle\hat{H}\rangle}{\partial n_{i \sigma}}=2\left\langle\varphi_{0}\right| \sum_{i k_{\|}}-2 t\left(\cos k_{x}+\cos k_{y}\right) \frac{\partial q_{i \sigma}}{\partial n_{i \sigma}} \hat{c}_{i k_{\|} \sigma}^{\dagger} \hat{c}_{i k_{\|} \sigma} \\
-\delta_{i, j \pm 1} \sqrt{\frac{q_{j \sigma}}{q_{i \sigma}}} \frac{\partial q_{i \sigma}}{\partial n_{i \sigma}} t\left(\hat{c}_{i k_{\|} \sigma}^{\dagger} \hat{c}_{j k_{\|} \sigma}+\hat{c}_{j k_{\|} \sigma}^{\dagger} \hat{c}_{i k_{\|} \sigma}\right) \sum_{i k_{\|}}\left|\varphi_{0}\right\rangle \\
-2 N_{k_{\|}} \lambda_{i \sigma}-2 N_{k_{\|}} \Lambda+\sum_{i} N_{k_{\|}} U \frac{\partial d_{i}}{\partial n_{i \sigma}}=0 \\
\frac{\partial\langle\hat{H}\rangle}{\partial \lambda_{i \sigma}}=\left\langle\varphi_{0} \mid \sum_{k_{\|}} \hat{c}_{i k_{\|} \sigma}^{\dagger} \hat{c}_{i k_{\|} \sigma} \sigma \varphi_{0}\right\rangle-N_{k_{\|}} n_{i \sigma}=0 \\
\frac{\partial\langle\hat{H}\rangle}{\partial \Lambda}=\left(N-2 N_{k_{\|}} \sum_{i} n_{\sigma}\right)=0 .
\end{gathered}
$$

In addition to the above equations, the electrostatic stability of the system should be considered by the inclusion of the Poisson equation. The effects of long-range electron-electron and electron-ion interactions on the electric potential could be evaluated by considering a simple version of the Poisson equation for slab geometry as follows: ${ }^{16}$

$$
v_{i}=v_{i}^{(0)}-\sum_{j, i \neq j} \alpha\left|z_{i}-z_{j}\right|\left(n_{j}-1\right),
$$

where $z_{i}$ is the position of plane $i$. Here, $\alpha=\frac{e^{2}}{2 a \epsilon_{0} \epsilon}$ where $\epsilon_{0}$ is vacuum permittivity, $\epsilon_{r}$ is relative permittivity, $a$ is lattice constant, and $e$ is electron charge. We considered three values of the Poisson coupling parameter $\alpha=0,0.02$, and 0.2 corresponding to $\epsilon_{r}=\infty, 150$, and 15, respectively. For $\alpha<$ 0.02 , the effect of the Poisson term on the relevant quantities is negligible, while for larger values, $\alpha>0.2$, additional screening occurs but we observe the same qualitative behavior. Experimental data show that for transition-metal oxides, the relative permittivity is usually very large. ${ }^{17}$ Therefore, because $\epsilon_{r}=\infty$ is a good approximation for strongly correlated materials, we choose $\alpha=0$ and neglect the additional screening of charges induced by the Poisson term.

In order to numerically solve the above set of nonlinear equations, we employ MINPACK.1, ${ }^{18,19}$ which uses a trustregion-dogleg method, while for the $k$-space summation, we choose a $16 \times 16$ Monkhorst-Pack ${ }^{20}$ grid, which gives accurate estimates for the total energy. From the above equations, it is obvious that the Jacobian matrix required by the nonlinear solver has to be calculated by a finite difference method because no analytical evaluation of the Jacobian matrix is possible. Also, note that the Jacobian matrix is dense and all of its elements are nonzero.

We also tried to implement another approach by solving Eqs. (15) and (19)-(22) iteratively by starting from an estimation of the variational parameters and a calculation of $\left|\varphi_{0}\right\rangle$, which are then supplied to the set of Eqs. (19)-(22) to find a new set of variational parameters and then the whole procedure is repeated. The iterative approach did not converge for values of $U>4 t$, which could be because of the high nonlinearity of the equations for large $U$. Other authors also reported similar problems with such an iterative scheme. ${ }^{21}$ In order to achieve convergence, it should be noticed that during the procedure of solving the set of Eqs. (19)-(22) to satisfy Eq. (21), one also needs to change $\left|\varphi_{0}\right\rangle$, otherwise there is no way to fix $\left\langle\hat{n}_{i \sigma}\right\rangle_{0}$ at $n_{i \sigma}$. Thus, it is possible in one step to fix $\lambda_{i \sigma}$ and $\left|\varphi_{0}\right\rangle$ and minimize with respect to $g_{i}, n_{i}$, and $\Lambda$, while in the next step to do the opposite. This speeds up the numerical procedure because there is no need to calculate $\left|\varphi_{0}\right\rangle$ for the changes of $g_{i}, n_{i}$, and $\Lambda$.

In the next sections, we report results for $q_{i}$ as the position-dependent QP weight, which is an indication of the mobility of the electrons in Fermi-liquid theory. It is possible to show that the inverse of this factor is proportional to the mass renormalization, which is divergent for $q_{i}=0$ and which corresponds to an insulating phase. ${ }^{22}$

The quantity $\tilde{v}_{i}=v_{i}+\lambda_{i \sigma}+\Lambda$ is considered as an effective potential, which acts effectively only on $\left|\varphi_{0}\right\rangle$. In the following, the parameters $U$ and $v$ are scaled with the tight-binding parameter $t$.

\section{B. Numerical results}

We solve the set of Eqs. (19)-(22) for a slab geometry and a linear distribution of the potential along the direction perpendicular to the slab in order to investigate its effect on the strong correlations.

The spatial distribution of the QP weights and the charge densities are shown in Figs. 1(a) and 1(b) and Figs. 2(a) and 2(b) for different values of the Hubbard repulsion $U$ for a slab of width $N_{z}=90$. The presence of a potential profile causes charge distortion in the system, and because of the nature of the Gutzwiller renormalization factors that are minimum at half-filling $\left(n_{i}=1.0\right)$, it is predicted that any deviation from half-filling may lead to larger QP weights when compared to the case without electric field.
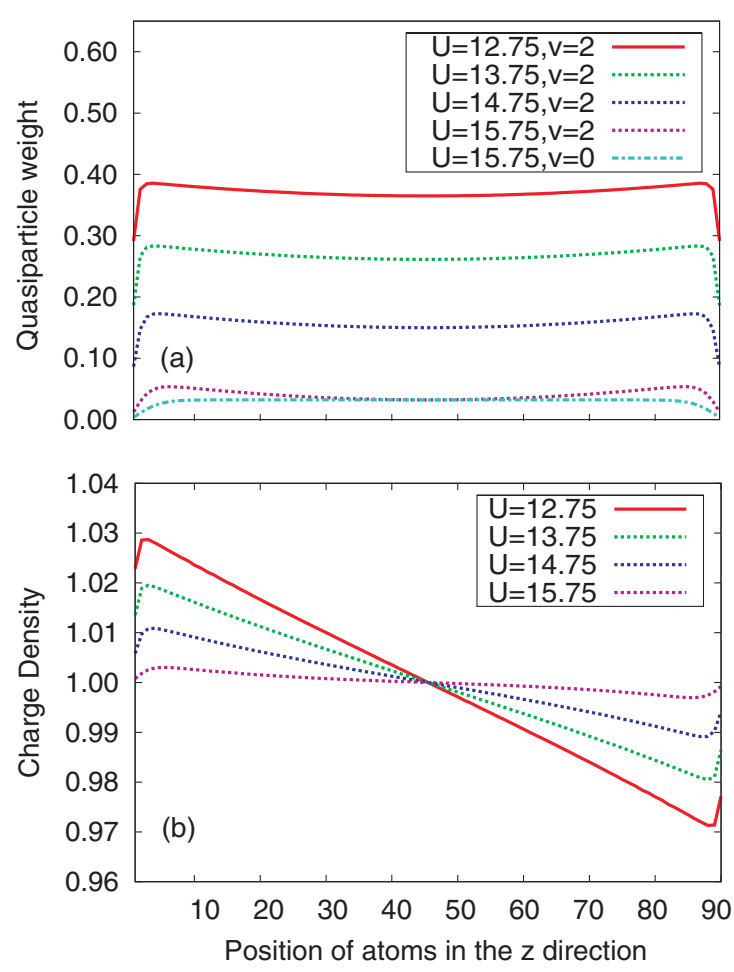

FIG. 1. (Color online) (a) QP weight distribution for $U<16$, $N_{z}=90$, and $v=2$ and 0; (b) charge distribution for $U<16, N_{z}=$ 90 , and $v=2$. Note that for $v=0$, the system is at half-filling $n_{i}=1$. 

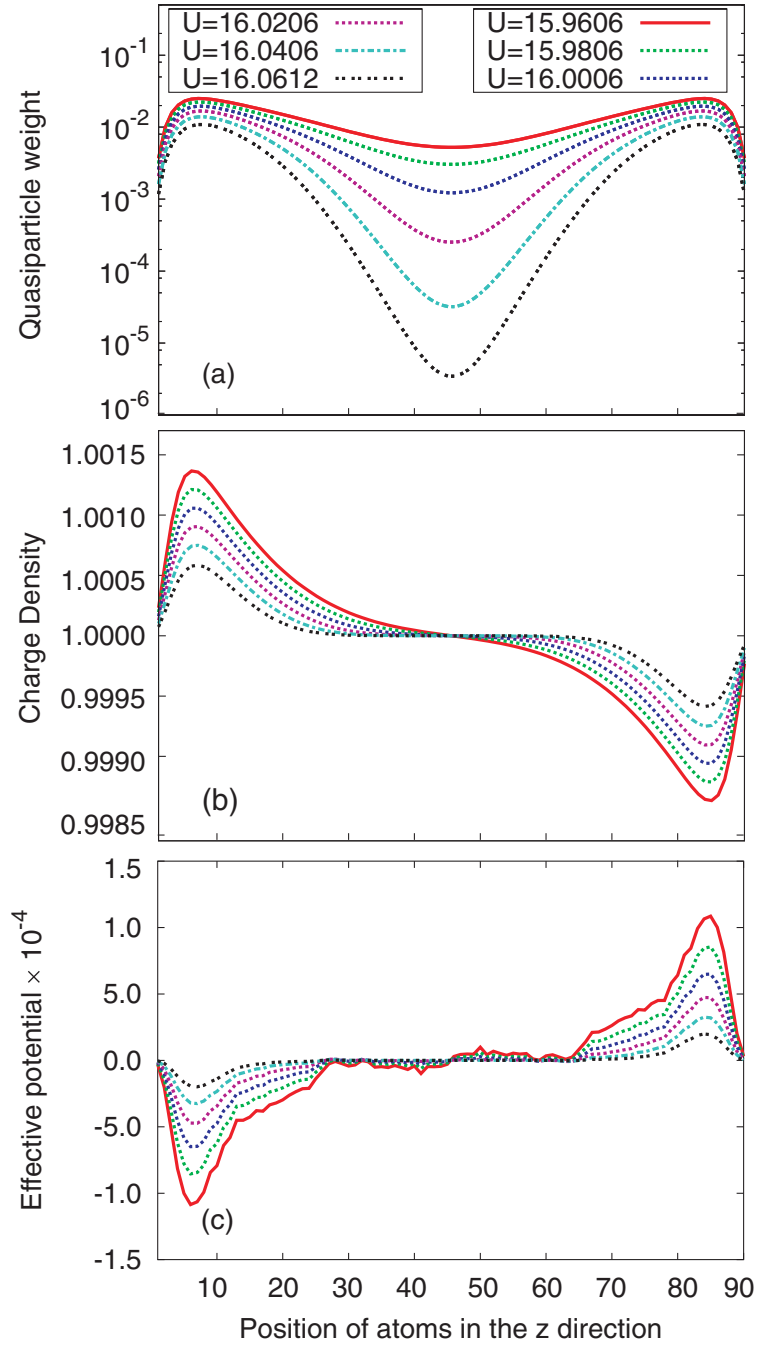

FIG. 2. (Color online) (a) QP weight distribution, (b) charge distribution, and (c) effective potential for $v=2$. Notice the $U / 2$ contribution is subtracted from the effective potential.

In the presence of an electric field, for both $U<U_{c}$ and $U>U_{c}$ (where $U_{c}=16$ for bulk), the maximum QP weight is achieved in few layers beneath the surface as is obvious from Figs. 1(a) and 2(a). For $U<U_{c}$ akin to the zero electric-field case, ${ }^{23}$ the minimum QP weight is achieved at the surface sites. In contrast, for $U>U_{c}$, the QP weight of the central atoms starts to dramatically drop to extremely low values and creates a dead region as is indicated in Fig. 2(a). This is presented more clearly in Figs. 3(a) and 3(b) where we show the QP weight versus the Hubbard repulsion for three significant locations (surface, near surface, and bulk) for both $v=0$ and 2 .

The formation of the dead zone leads to charge being trapped near the surfaces of the slab because the tunneling through the bulk is suppressed. This charge trapping prevents the system to exhibit a metal-insulator transition even for values of the Hubbard repulsion larger than the bulk $U_{c}$. This result is contrary to the zero-field case where for $U$ close to $U_{c}$, the surface region forms a dead zone instead of the central region. Therefore, in the absence of an electric field, the QP weight is maximal in the central parts as shown in Fig. 1(a). This is due to the fact that the surface sites have a
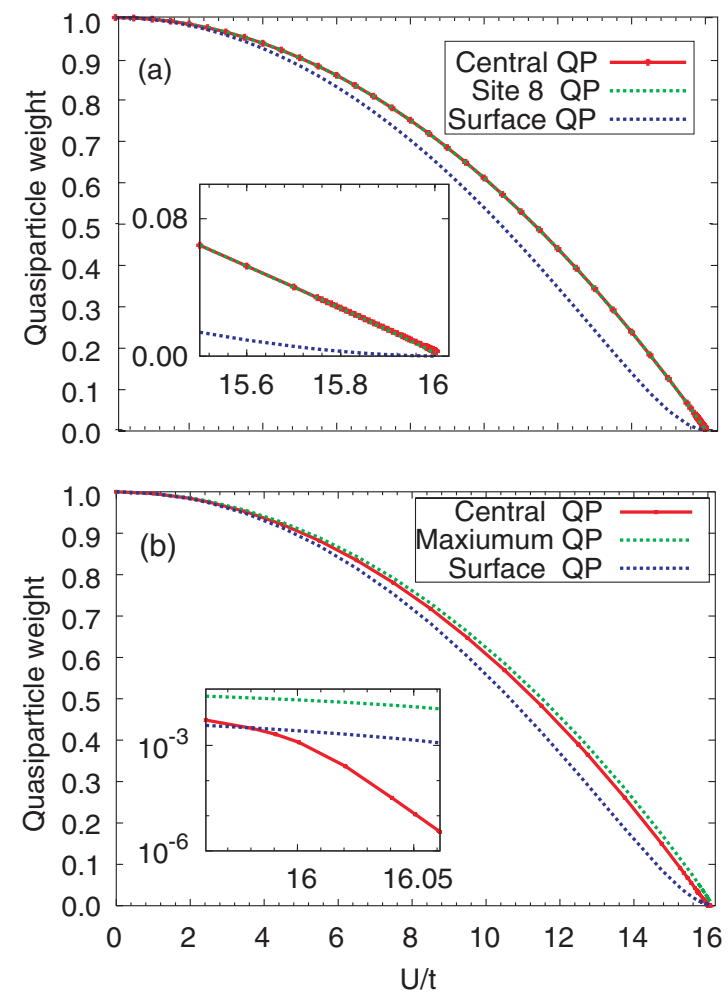

FIG. 3. (Color online) QP weight of various sites versus Hubbard repulsion for $N_{z}=90$, (a) $v=0$, and (b) $v=2$.

lower kinetic energy (due to lower coordination number at the surface). Interestingly, surface sites will always have a finite (but low) QP weight, as long as the bulk QP weight is finite. The surface sites are always able to gain kinetic energy from the central sites that have the highest QP weight, which act as a source of kinetic energy. ${ }^{23-25}$

In Figs. 2(b) and 2(c), the spatial distribution of the charge densities and the effective potential are shown for different values of $U>U_{c}$. Both of these two quantities behave similarly to the QP weight. The charge density is maximum in the same location in which we have the maximum QP weight, while for the sites with charge density near local half-filling $\left(n_{i}=1.0\right)$, we have the lowest QP weight and this is where the electric field has the weakest effect. This confirms that the larger QP weight is due to a larger carrier density near the surface of the slab. The deviations of the carrier densities from half-filling correspond to larger electron density for sites with lower effective potential and hole density for sites with higher effective potential as shown in Fig. 2(c). The charge frustration is responsible for the nonzero QP weight for these sites near the surfaces of the system even for $U>U_{c}$.

Figure 4 shows the change of QP weight throughout the system as the voltage difference is increased for $U=16.0602$. Notice that the location of the maximal QP weight slowly shifts toward the surface and at the same time its value increases with electric field. As a consequence, the size of the central dead zone reduces with increasing applied voltage difference. One should note that when measuring an $I-V$ curve, only the in-plane conductivity will exhibit metallic behavior because the $z$-axis conductivity will be dominated by the bulk insulating layer. 


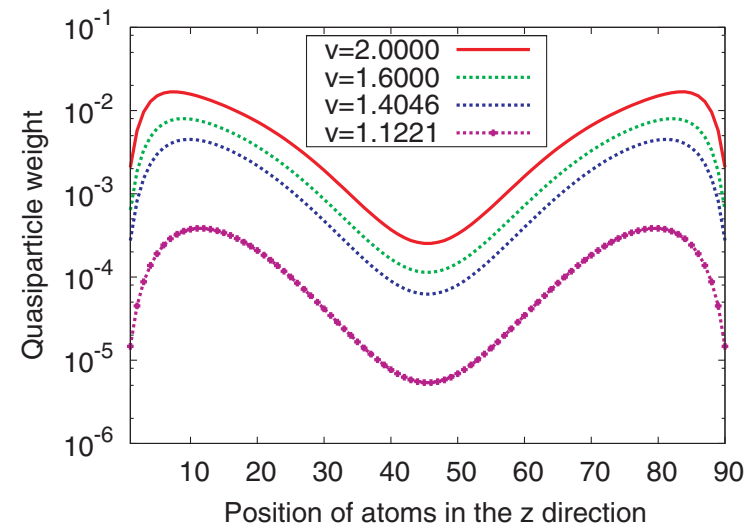

FIG. 4. (Color online) QP weight distribution for various potential difference for $U=16.0602$ where $N_{z}=90$.

In order to better understand the formation of the dead zone with suppressed QP weight, we plot in Fig. 5 the dependence of the central QP weight on the thickness of the slab. This is shown for two values of the potential difference, $v=1.8$ and 2 , and for two values of the Hubbard repulsion, above and below the critical $U_{c}, U=15.75$ and 16.025, respectively. As discussed previously, in the presence of an electric field, sites near the surface will always have larger QP weight due to the accumulation of surface charge. This will ensure that the central region will always have its QP weight enhanced due to its proximity to regions with increased kinetic energy. As shown in Fig. 5, we can distinguish two regimes, depending whether the Hubbard repulsion is above or below the critical value obtained in the absence of the electric field. When $U<$ $U_{c}$, the QP weight of the central sites converges toward a finite value since even in the absence of the electric field, the system is still metallic, albeit with a small QP weight. When $U>U_{c}$, the homogeneous system should be insulating with vanishing QP weight. Instead, even if the electric field is screened in the central region, the QP weight will never exactly vanish because it is in contact with a doped region with finite QP weight. We can infer that for large enough electric fields and as long as the slab is finite, the QP weight will never vanish.

Next, we discuss the possible appearance of a critical field value above which the system is metallic. We plot in Fig. 6(a) the maximum and central QP weights for four values of $\delta U=$ $U-U_{c}$ as a function of potential difference $v$. Note that for



FIG. 5. (Color online) QP weight at the center of the slab versus width for $v=1.8,2$, and two values of the Hubbard repulsion $U=$ $15.75,16.025$.
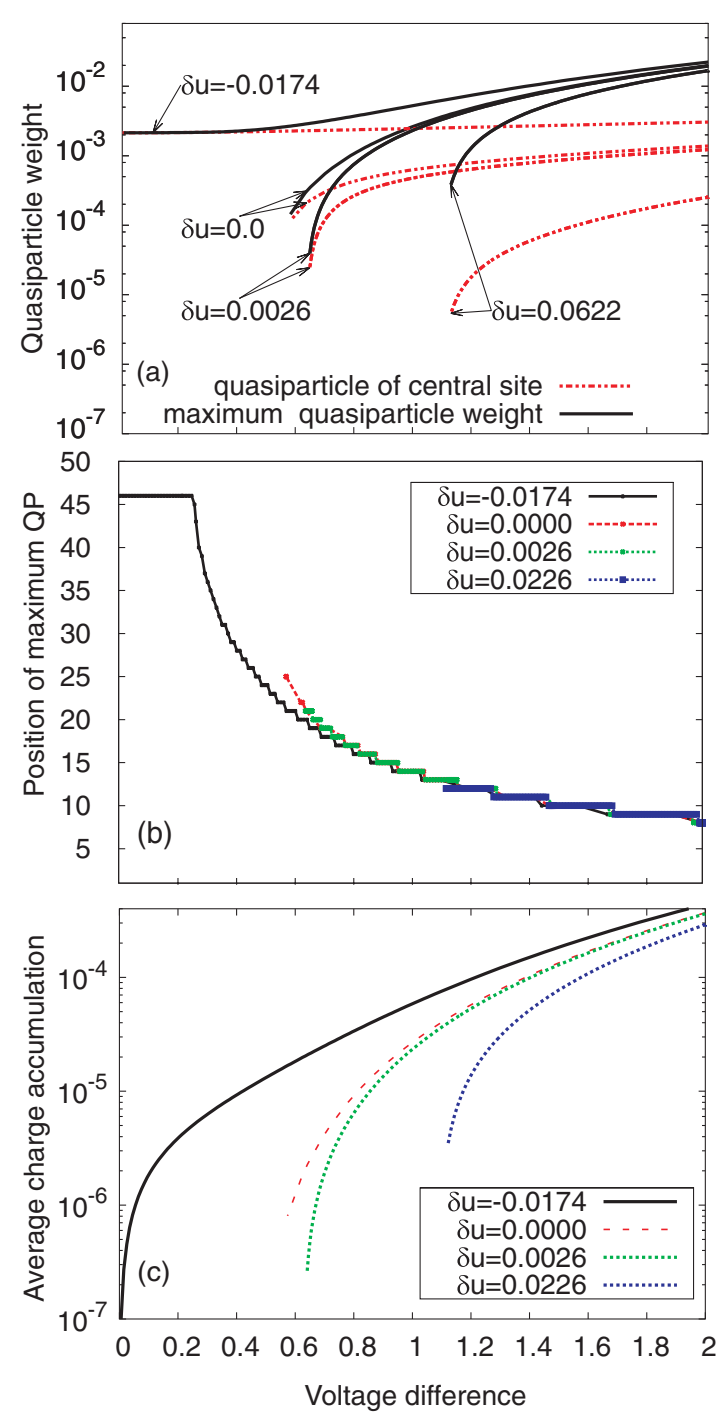

FIG. 6. (Color online) (a)Maximum and central QP weight, (b) position of maximum QP weight, (c) average charge accumulation versus $v$ for $\delta U=U-U_{c}=0.0226,0.0026,0.0$, and -0.0174 where $N_{z}=90$.

larger Hubbard repulsions, the minimization procedure is not stable if the maximum QP is strongly suppressed, and thus we have converged solutions only above certain field strengths. We observe that as the electric field increases, the difference between the maximal and central QP weights increases in all situations since the maximum will be near the surface. This can be also seen in Fig. 6(b), where the location of the maximum QP weight is plotted as a function of electric field. For low-field values, the maximum is located at the center of the slab not only due to the vanishing charge accumulation near the surface [shown in Fig. 6(c)], but also due to the increase in the correlation length near half-filling. Although the electron density might have a local maximum near the surface due to the presence of the electric field, a long correlation length will ensure that the suppression due to the existence of the surface recovers only deep into the central regions.

It is important to note that for $\delta U=-0.0174$, the QP weight recovers its bulk value when the electric field goes to zero, while for $\delta U>0.0$, it decreases rapidly at a finite critical 


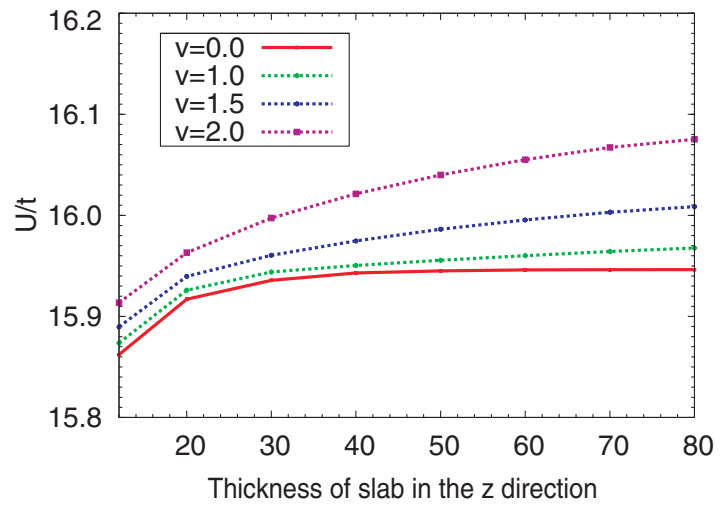

FIG. 7. (Color online) Critical Hubbard repulsion for which the maximal $\mathrm{QP}$ weight is $Z=5.0 \times 10^{-3}$ versus slab thickness for different electric fields.

$v_{c}$. Although our method breaks down when the QP weight is very small, the results hint toward a true metal-insulator phase transition when the Hubbard repulsion totally screens the electric field. To accurately settle whether at $v_{c}$ there is a rapid increase of the QP weight or a true phase transition, either analytical approaches, arbitrary precision methods, or DMFT calculations below $v_{c}$ are necessary.

Next, we discuss the effect of slab dimensions and in Fig. 7 we show as a function of thickness the value of the Hubbard repulsion for which the maximum QP weight is $Z=5.0 \times 10^{-3}$. This will give a lower bound for the critical $U_{c}^{\text {slab }}$ in the presence of a perpendicular electric field. We observe that $U_{c}^{\text {slab }}$ is larger for larger thicknesses and stronger fields $v$. Again, this is related to the amount of charges localized near the surfaces. When $U$ increases, the QP weight corresponding to the central parts drops faster in the thicker slabs, as is indicated in Fig. 8(a), and this causes more charge accumulation at the surfaces. This happens because the probability of the electrons to tunnel through the central parts is being reduced, which makes charge relaxation more difficult. In other words, by increasing the Hubbard repulsion, the system tries to screen the charges in order to lower the energy, while on the other hand, the increase of $U$ suppresses the metallic behavior of the central part and thus hinders the charge relaxation. This can be better understood by considering the average charge accumulation in half of the slab, which increases slowly with the slab's thickness as indicated in Fig. 8(b). The numerical calculations indicate that even for $U>U_{c}$, we did not obtain a clear asymptotic behavior for maximum QP weight by increasing the slab width. One may expect that an asymptotic solution is reachable for thicker slabs, but this turns out to be beyond our numerical resources.

\section{CONCLUSIONS}

In conclusion, we described the Mott metal insulator in a slab geometry in the presence of a perpendicular external electric field by calculating the site-dependent QP weight. This is done by using an inhomogeneous Gutzwiller approximation, which is exact in the limit of infinite dimensions. Increasing the Hubbard repulsion in the presence of an external electric field

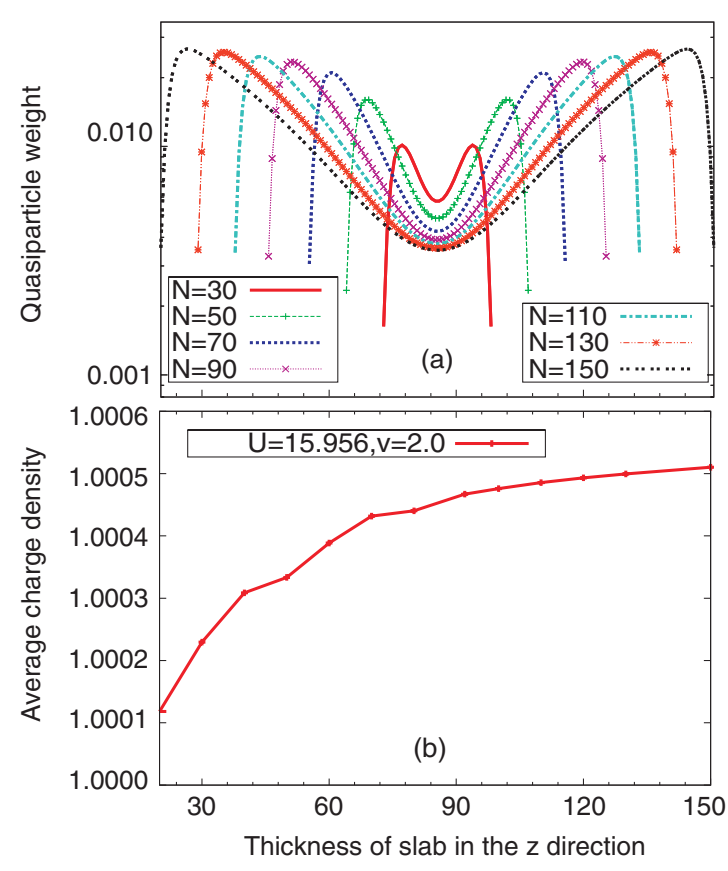

FIG. 8. (Color online) (a) QP weight distribution of different sites for various slab thicknesses. (b) The charge density averaged over half of the slab for different thicknesses. Here, $U=15.956$ and $v=2$.

leads to the formation of a dead insulating zone at the center of the thin film. The formation of the dead zone for $U>16$ occurs before complete screening of the electric field and, therefore, charge trapping occurs at the surface. This causes the MIT to be shifted in the presence of the external field. We therefore show that even though the QP weight of the central region is strongly suppressed for $U>U_{c}^{(\text {bulk })}$, the surface layers remain metallic and with larger QP weight.

Although our calculation can not give a definitive answer on whether a critical value $v_{c}$ of the electric field exists, below which the slab becomes insulating, it shows that a rapid change in the maximum QP weight and charge accumulation

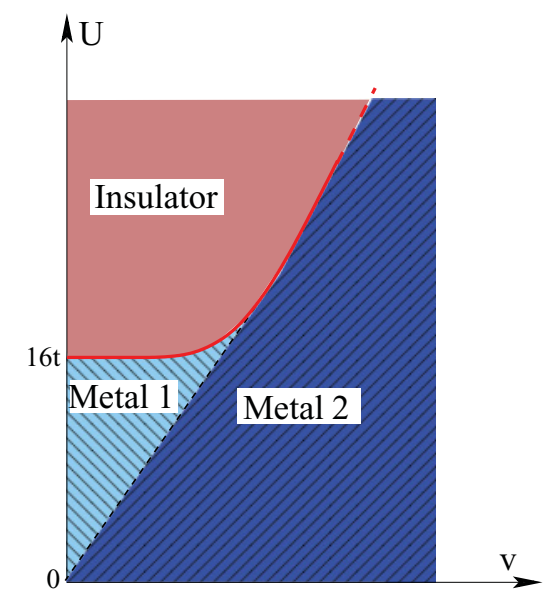

FIG. 9. (Color online) Sketch of the possible electric-fieldinduced changes on the phase diagram. The two metallic regions differ only in the location of the maximum QP weight: center of slab in region 1 and near surface in region 2 . 
will occur above $v_{c}$. The resulting phase diagram is sketched in Fig. 9 and shows the electric-field-induced shift of the metal-insulator transition. We uncover two metallic regions, depending whether the maximum QP weight is achieved in the center of the slab (region 1) or near the surface (region 2). Analytical or numerical methods, which can accurately probe the insulating region, will shine light on the exact nature of this transition.

From an experimental point of view, our results are relevant for transport measurements in thin films. In the presence of an external electric field perpendicular to an insulating film, one could use the surface states for transport since the charge transfer at the surface creates two-dimensional underdoped and overdoped regions. In the same time, transport perpendicular to the thin film is suppressed due to the dead insulating zone, thus protecting the surface states from leakages. The electric field needed to create the surface states is also much lower than the breakdown field needed to pass current across the insulating zone.

\section{ACKNOWLEDGMENT}

This work was supported by the Flemish Science Foundation (FWO-Vlaanderen) and the Belgian Science Policy (IAP).
${ }^{1}$ A. Cavalleri, Th. Dekorsy, H. H. W. Chong, J. C. Kieffer, and R. W. Schoenlein, Phys. Rev. B 70, 161102(R) (2004).

${ }^{2}$ Hyun-Tak Kim and Byung Chae, New J. Phys. 6, 52 (2004).

${ }^{3}$ A. D. Caviglia, S. Gariglio, N. Reyren, and D. Jaccard, Nature (London) 456, 624 (2008).

${ }^{4}$ D. Ruzmetov, G. Gopalakrishnan, and J. Deng, J. Appl. Phys. 106, 083702 (2009).

${ }^{5}$ A. Husmann, J. Brooke, T. F. Rosenbaum, X. Yao, and J. M. Honig, Phys. Rev. Lett. 84, 2465 (2000).

${ }^{6}$ J. Hubbard, Proc. R. Soc. London 276, 238 (1963).

${ }^{7}$ M. C. Gutzwiller, Phys. Rev. Lett. 10, 159 (1963).

${ }^{8}$ W. Metzner and D. Vollhardt, Phys. Rev. Lett. 62, 324 (1989).

${ }^{9}$ W. Metzner and D. Vollhardt, Phys. Rev. B 37, 7382 (1988).

${ }^{10}$ F. Gebhard, Phys. Rev. B 41, 9452 (1990).

${ }^{11}$ E. Lieb and F. Y. Wu, Phys. Rev. Lett. 20, 1445 (1968).

${ }^{12}$ G. Kotliar and A. E. Ruckenstein, Phys. Rev. Lett. 57, 1362 (1986).

${ }^{13}$ A. Georges, G. Kotliar, W. Krauth, and M. J. Rozenberg, Rev. Mod. Phys. 68, 13 (1996).

${ }^{14}$ J. Bunemann, F. Gebhard, and R. Thul, Phys. Rev. B 67, 075103 (2003)
${ }^{15}$ W. F. Brinkman and T. M. Rice, Phys. Rev. B 2, 4302 (1970).

${ }^{16}$ R. Nourafkan and F. Marsiglio, Phys. Rev. B 84, 075133 (2011).

${ }^{17}$ P. Lunkenheimer, S. Krohns, S. Riegg, S. G. Ebbinghaus, A. Reller, and A. Loidl, Eur. Phys. J. Special Topics 180, 61 (2010).

${ }^{18}$ J. J. More, B. S. Garbow, and K. E. Hillstrom, Argonne National Laboratory Report No. ANL-80-74, 1980 (unpublished).

${ }^{19}$ J. J. More, D. C. Sorensen, K. E. Hillstrom, and B. S. Garbow, in Sources and Development of Mathematical Software, edited by W. J. Cowell (Prentice-Hall, Englewood Cliffs, NJ, 1984), pp. 88-111.

${ }^{20}$ H. J. Monkhorst and J. D. Pack, Phys. Rev. B 12, 15 (1976).

${ }^{21}$ A. Ruegg, S. Pilgram, and M. Sigrist, Phys. Rev. B 75, 195117 (2007).

${ }^{22} \mathrm{P}$. Fazekas, Lecture Notes on Electron Correlations and Magnetism, Series in Modern Condensed Matter Physics, Vol. 5 (World Scientific, Singapore, 1999).

${ }^{23}$ R. Nourafkan and F. Marsiglio, Phys. Rev. B 83, 155116 (2011).

${ }^{24}$ M. Potthoff and W. Nolting, Phys. Rev. B 59, 2549 (1999).

${ }^{25}$ G. Borghi, M. Fabrizio, and E. Tosatti, Phys. Rev. B 81, 115134 (2010). 